\title{
Recovery Focused Behavior Therapy in Treatment of Obsessive-Compulsive Disorder: A Case Study
}

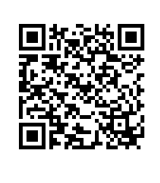

\author{
Tarana Jain* and Rakesh Jain \\ Institute of Mental Health and Hospital, India
}

Submission: February 06, 2018; Published: April 10, 2018

*Corresponding author: Tarana Jain, Institute of Mental Health and Hospital, India, Email: tarana.jain@gmail.com

\begin{abstract}
Obsessive Compulsive Disorder (OCD) is one of the most prevailing disorders in our society. It is treatable with combined management of pharmacotherapy and psychotherapy. Exposure and Response Prevention is documented as the most successful psychotherapy in treating OCD patients efficiently. However, it is also observed that such procedures could induce immense amount of distress in the individuals. Some non-aversive forms of psychotherapies like cognitive-restructuring are also being developed to manage the patients who get overwhelmed with the exposure therapies. We applied a minimal aversive form of behavioral intervention, which is labeled as Recovery Focused Behavior Therapy (RFBT) in a case of OCD, predominantly compulsive type. Recovery Focused Behavior Therapy is a gentle approach in treating OCD cases, which focuses on the future of how the patient would actually present adaptive responses after being fully recovered from the ailment. The case presented to us had intrusive, recurrent, uncontrollable and persistent concerns regarding dirt. This led to an increased pathological anxiety in the patient and to reduce this anxiety he would engage in repetitive and ritualistic acts of cleaning all the objects that he touched, washing hands and bathing for countless hours. We treated him for 6 sessions including follow-ups. In RFBT, our primary focus was to increase the adaptive behavior in the patient. Consequently, this approach resulted in tremendous improvement in this case. Further studies will be required to completely understand the mechanisms and applications of such cases.
\end{abstract}

Keywords: Obsessive Compulsive Disorder; Recovery Focused Behavior Therapy; Exposure Response Prevention; Cognitive Behavior Therapy; Cognitive Drill Therapy

Abbreviations: OCD: Obsessive Compulsive Disorder; RFBT: Recovery Focused Behavior Therapy; Y-BOCS: Yale-Brown Obsessive Compulsive Scale

\section{Introduction}

Obsessive Compulsive Disorder (OCD) is one of the most crucial mental illnesses prevailing in our society. Jaisoorya et al. [1] reported a point prevalence of $3.3 \%$, with a prevalence rate of $3.5 \%$ in males and $3.2 \%$ in females in Indian sample. Moreover, $8.5 \%$ of Indian students had sub threshold OCD. In the same study, they found that $67.1 \%$ had taboo thoughts, whereas $57.4 \%$ engaged in mental rituals. In another study [2] found that $60 \%$ of the clinically diagnosed OCD cases had contamination fears, with $63.63 \%$ males and $57.14 \%$ females. Fortunately, in today's scenario, this disorder is treatable with promising results from evidence-based approaches such as Exposure and Response Prevention, which was developed in the 1960s. It is the most popular treatment opted by many therapists and its efficacy is highly supported by many researches as a first line treatment [3]. Moreover, nowadays a huge emphasis is also paid to the combined approach with pharmacotherapy and CBT, which encompasses ERP as a first line of treatment for OCD [4]. There are also many new forms of psychotherapy that are being developed to address the key role played by the cognitive factors in the maintenance of OCD, such as cognitive behavioral therapy and cognitive drill therapy [5].

A planned or accidental exposure to feared cues triggers anxiety and obsessions in afflicted individuals. The experience of anxiety includes aversive somatic experience in the form of physiological symptoms such palpitations, sweating, dry mouth, butterfly in the stomach etc. These body reactions are aversive to the patient, which leads to operant behaviors of compulsive acts, which negatively reinforce the OCD behaviors. There are patients who find exposure therapies as overwhelming and even refuse to undergo it. To address this issue cognitive-restructuring was developed with more or less success [6]. Still other approaches like mindfulness based meditation [7], Acceptance-Commitment Therapy [8] are also being tried in cases of OCD. Kumar \& Sahu [9] tried RFBT in a case of OCD. We tried the same approach in contamination OCD, which yielded desired success. 


\section{Case Summary of the Patient}

A 58 years old married male, belonging to urban area presented with chief complaints of constant preoccupation regarding dirty objects, spending lot of time in washing hands and bathing, fears that he will get infected and that he will fall ill if he does not wash himself, headache when anxious, and decreased sleep. His illness spanned for a total duration of 8 years. The index patient was asymptomatic until 8 years back, when it was noticed that he constantly does hand washing throughout the day after touching the tap or washbasin, taking long hours to shower and bathing again if his any part of the body touched the wall again, soon after the shower. If someone's shoes were kept on his shoes, then he had to wash his feet and shoes before wearing it. If a dog passes nearby, he had to take a shower fearing that he might be infected from the dog. If someone has come from outside and kept their bags on the bed, then he would wash the bed sheets as it might have been contaminated by the bag. His constant fears were that if he didn't engage in these washing rituals, then he felt that he would become dirty and untouchable, that dirt would spread all over the house, others would fall ill in the family and eventually they all would die. In 2010, he consulted a psychiatrist; however, no improvement was seen, although his compliance to medicines was good. Moreover, OCD resulted in dysfunction in his personal and social life to such an extent that he was contemplating suicide. It was at this point of time when his son brought him for psychological treatment.

\section{Baseline on outcome measures}

Yale-Brown Obsessive-Compulsive Scale (Y-BOCS) severity scale was administered. His score was 30 out of maximum 40 indicating severe form of OCD. Sheehan Disability Scale was administered to assess psychosocial disability due to OCD. His score was 21 indicating high levels of psychosocial disability in the areas of work, social and family life attributable to OCD.

\section{OBSD analysis}

An OBSD analysis Jain $[10,11]$ was conducted to identify objects that are associated with OCD, body-mind reactions elicited by an exposure to the objects of anxiety, safety behaviors in the form of avoidance and compulsions and the perceived feared outcomes. The analysis revealed following picture:

\section{Objects of OCD}
a. Touching the tap and wash basin
b. Touching the wall after bathing
c. Others shoes touching his shoes
d. Someone keeps bag on bed
e. A street dog passes by

\section{Body-mind reactions}
a. Sweating
b. Heart palpitation
c. Headache
d. Decreased concentration

\section{Safety-Behaviors}
a. Washing hands 5 to 6 times
b. Bathing again
c. Has to wash feet and shoes before wearing it
d. Washing bed sheets
e. Bathing after the street dog passes by

\section{Danger Ideations}
a. Germs will spread whatever I touch
b. I have got infected again
c. My feet will get dirty and I will get infected with germs
d. If I don't wash then the germs will spread and harm others too

e. The dog must be carrying germs, I will fall sick if I don't bathe now.

It was noticed that his objects of OCD were linked with imaginary fear of harm, which contained future time perspective and did not cause any immediate danger to him. The objects of OCD were triggering heightened anxiety and to reduce it, the patient was engaging in compulsive neutralizing behaviors.

\section{Recovery focused behavioral treatment}

RFBT focuses explicitly on contemplation and emission of healthy adaptive behaviors in the situations and contexts that currently elicit anxiety response and subsequent avoidance and compulsive behaviors. The patient is required to imagine a future timeframe, which is symptom free and emit adaptive behaviors in the domains of feelings, thoughts and acts. Typical protocols of RFBT questions are "how would you touch a dirty object without any anxiety when you are symptom free?", "What would you think of dirty objects when you are symptom free?" Can you show me how would you use dirty objects without anxiety and compulsion when you are symptom free?". The patient is required to report verbally the feelings, thoughts and acts that he/she would emit upon recovery; also to repetitively demonstrate such adaptive behaviors to the clinician and continue to emit similar behaviors as home assignment. If there is any activation of anxiety, the patient is reminded that since he is having problems now he is experiencing anxiety reaction, but when he is recovered there would be no anxiety reaction. A session lasts for about 45 minutes. Detailed session wise work up done with this patient is summarized below: 
Session 1: Building the therapeutic alliance, assessment and Psycho-education: The rapport with the patient was easily established. Thereafter, relevant case history was collected. Y-BOCS and Sheehan Disability Scale were administered as outcome measures. He was psycho-educated regarding his problem of OCD and role of anxiety, compulsive behaviors and danger ideations in the maintenance of the disorder. Also he was given a treatment rationale that he needed to repetitively imagine how he wants to behave after recovery when exposed to dirty objects. An orientation to OBSD analysis was given that objects of OCD elicit body-mind reactions and danger ideations for which he engages himself in safety behaviors. His task would be to learn to handle contaminated objects without any anxiety response.

Session 2: Commencement of RFBT: The session commenced with a brief preview of the previous session. It was noticed that he clearly understood the nature and cognitive-behavioral determinants of his problem. He was told that the nature of his danger perceptions were future oriented as he was anticipating harm somewhere in future which was leading to his anxiety symptoms. Thus, he was told that an effective way to overcome this threat was to imagine how he would feel, think and react when he would be symptom free. Various dirty objects were identified and exposure was planned from low to high anxiety provoking stimuli. Low anxiety-provoking dirty objects were placed before him and modeling of desired behaviors was done by the clinician. A few objects were picked up and demonstrate with simultaneous explanations that upon recovery how would he be touching and picking up dirty objects in his hands smoothly and easily without any discomfort and fear of harming others. He was ensured that he would remain calm and comfortable by holding such objects. Thereafter, he was asked to imagine how he would pick up these objects and stay calm once he was symptom free. When he confirmed that at covert level he could stay calm by holding the dirty objects; he was required to demonstrate how actually he would be touching and picking these dirty objects.

One by one he picked up all low anxiety-provoking dirty objects. The RFBT rationale was repeated intermittently for emphasis. It was highlighted that after recovery he would be comfortable to deal with dirtier objects, which provoke high anxiety, in similar manner. Subjective Reports of Discomforts were obtained on Visual Analogue Scales intermittently. He reported little discomfort for modeled behavior, his own covert behavior and actual handling of low anxiety-provoking dirty objects. More covert and overt exercises were carried out for other objects like touching blacking spots on the chair, touching clothes, touching walls, curtains, windows, table, etc. He was also instructed to imagine washing hands normally as and when required. Whenever, there was an activation of high anxiety during this exposure, he was reassured that he is experiencing the symptoms in present time, but there would be no anxiety symptoms when he would touch these objects when he would be symptom free. He was told to retain his focus on the future and perceived symptom free time. After the exposure, within session report was obtained. He was feeling much better and light. Thereafter, a homework assignment was given to him to practice the same at home with which he readily complied to.

Session 3: Reducing the compulsions with the help of RFBT: The session commenced with a brief preview of the previous session. The patient reported that he felt 25 percent better than before. He reported a decline in his constant hand washing behavior, the usage of water had decreased and his sleep had improved. He also reported that he had problems walking bare feet and when a dog would slightly touch him, he would be compelled to take a bath. He said that he had generalized the treatment to these situations, resultantly feeling more confident and relaxed during the same. After receiving a positive feedback, this treatment was continued for the remainder of the objects of OCD. This time, the targets were situations such as a dirty shoe accidently kept over his shoe and a dirty bag kept on bed etc. He was required to imagine the symptom free time in future and how would he handle these objects.

After the exposure, the patient reported an improvement in his anxiety and said that he was feeling much better and light. Thereafter, a homework assignment was given to him to practice the same at home with which he readily complied to.

\section{Session 4: Targeting more objects of OCD using RFBT}

In this session, the patient was visibly much happier and better than before. This observation further coincided with patient's report that he felt that his mood was uplifted and that now he liked mingling with others. Moreover, his suicide ideations had declined as well. He marked his improvement as $70 \%$, the left over $30 \%$ was lingering over the issue of walking bare feet. The patient had still reported an increased frequency of washing his feet. This issue was addressed by giving another assignment of RFBT. He was asked to imagine how he would walk bare feet on a dirty surface after he was symptom free. He narrated a scene regarding how he would easily walk without any fear of feet getting dirty. Gradually, as the drill progressed he reported reduced physiological reactions and reported comfort with the idea of walking bare feet without continuously washing it. At this point of time another Y-BOCS severity measurement was done and the ratings had drastically dropped to 12 . The patient was given homework until the next session.

Session 5: Enhancing adaptive responses in the patient: In this session, the patient reported a drastic improvement from the previous symptoms. He said that he would no longer be worried about walking bare feet and he felt very happy. His severity ratings on YBOS had reduced to 7 . At this juncture, the patient was educated regarding healthy hygiene habits as well. He was taught to demarcate between normal levels of washing 
and exaggerated levels of washing. The patient was then given homework and called after a period of one month for follow up.

Session 6: Follow-up sessions to ensure the maintenance of improvement: The patient was seen after a period of one month. It was noted here that the patient was strictly complying with his homework, which led to maintenance of his improvement. His personal and social life had drastically enhanced. There were signs of great deal of relief in his appearance and behavior. The Y-BOCS scores were maintained at 7, whereas the work, social and personal disability had significantly decreased to 8 on Sheehan Disability Scale. Here the patient was asked to keep adhering to his medications and his therapy homework.

\section{Mechanisms underlying the successful outcome of RFBT}

The following mechanisms are considered to underlie the successful outcomes in RFBT.

Motivation to change: The patient had a very high motivation to change, as the illness was highly distressful and disabling for him.

Time reference: When a person experiences anxiety, it is always with respect to future harm, that is, there is no immediate danger being experienced by the individual, however the anticipation of the danger is distressing for him. This anticipation of the danger disables him to carry on an adaptive response in the present, which is already known by the patient. Hence, the focus is to highlight the adaptive responses to the feared stimulus that the individual would exhibit in future when asymptomatic.

Adaptive Responses: The rationale here is that, the rational and adaptive behavior is already present in the individual. However, it is buried under an immense amount of pathological anxiety. Hence, when the statement says that "when you will be recovered, how will you carry out a certain task", is to enhance the rational mind that is not link to any feared consequences or danger ideations. Following such adaptive responses effectively would lead to relapse prevention.

Gentle exposure: The patient was exposed to the anxietyprovoking situation however; the resultant intense anxiety was controlled as the patient was asked to imagine a future scenario of dealing with the situation when he was healed.

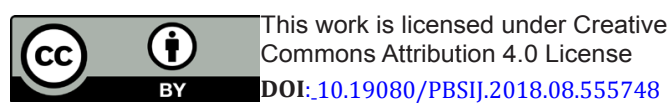

Response Prevention: Since a gentle exposure was given and a situation was asked to imagine wherein a task would be adaptively performed, the patient automatically did not indulge in any compulsive behaviors, thus preventing maladaptive responses.

\section{Conclusion}

The present case showed remarkable improvement with Recovery Focused Behavior Therapy, which was maintained over time without experiencing any excruciating anxiety, hence we termed this as a gentle therapy for OCD. However, further cases would be required to fully understand the mechanisms and efficacy of this therapy.

\section{References}

1. Jaisoorya TS, Reddy YCJ, Nair BS, Rani A, Menon PG, et al. (2017) Prevalence and Correlates of Obsessive-Compulsive Disorder and Subthreshold Obsessive-Compulsive Disorder among College Students in Kerala. Indian J Psychiatry 59(1): 56-62

2. Rajashekharaiah M, Verma P (2016) Phenomenology of obsessions and compulsions in Indian patients. Indian Journal of Contemporary Medical Research 3(7): 2139-2143.

3. Mc Kay D, Sookman D, Veale D (2014) Efficacy of cognitive behavioral therapy for obsessive compulsive disorder. Psychiatry Res 225(3): 236-246.

4. Gupta S, Wadhawan JM (2013) A Comparative Study of Management of Obsessive Compulsive Disorder: Pharmacotherapy Combined with Cognitive Behavioral Therapy versus Pharmacotherapy alone. Delhi Psychiatric Journal 16(1): 15-20.

5. Kumar R, Sameer A, Singh B (2012) Preliminary test of cognitive drill as an intervention for Obsessive Compulsive Disorder. Indian Journal of Clinical Psychology 39(1): 67-74.

6. Salkoviskis $\mathrm{P}$ (1999) Understanding and treating Obsessive Compulsive Disorder. Behav Res Ther 37(1): 29-52.

7. Key BL, Row K, Bieling P, Mc Cabe R, Pawluk EJ (2017) Mindfulness based cognitive therapy as an augmentation treatment for obsessivecompulsive disorder OCD. Clin Psychol Psychother 24(5): 1109-1120.

8. Vakili Y, Gharraee B (2014) The Effectiveness of Acceptance and Commitment Therapy in treating a case of OCD. Iran J Psychiatry 9(2): 115-117.

9. Kumar R, Sahu R (2017) Recovery focused behavior therapy: A case study. J Proj Psy Ment Health 24: 64-68.

10. Jain R (2016) Cognitive Drill Therapy.

11. Kumar S, Rakesh J (2008) Institute of mental health and hospital, Agra: Evolution in 150 years. Indian J Psychiatry 50(4): 308-312.

Your next submission with Juniper Publishers
will reach you the below assets
- Quality Editorial service
- Swift Peer Review
- Reprints availability
- E-prints Service
- Manuscript Podcast for convenient understanding
- Global attainment for your research
- Manuscript accessibility in different formats
( Pdf, E-pub, Full Text, Audio)
- Unceasing customer service
Track the below URL for one-step submission
https://juniperpublishers.com/online-submission.php

